\title{
SÍNDROME METABÓLICA E EDUCAÇÃO FÍSICA ESCOLAR
}

Gustavo Monnerat Cahli, Universidade Federal do Rio de Janeiro - UFRJ, Rio de Janeiro - Brasil

Thiago Teixeira Guimarães, Universidade Federal do Rio de Janeiro - UFRJ, Rio de Janeiro - Brasil

Alexandre Palma de Oliveira, Universidade Federal do Rio de Janeiro - UFRJ, Rio de Janeiro - Brasil

\section{RESUMO}

Síndrome metabólica é a designação atribuída a um conjunto de alterações fisiológicas no metabolismo humano que aumenta drasticamente o risco de desenvolver doenças cardiovasculares e diabetes, patologias que são as principais causas de morbidez e mortalidade atualmente. Um dos grupos frequentemente atingidos é o de crianças e jovens em idade escolar. Diversos estudos epidemiológicos e biológicos sugerem que a prática de atividades físicas pode melhorar significativamente esse quadro clínico, sendo uma importante ferramenta para o seu controle e tratamento, gerando saúde, menos sofrimento e redução de gastos públicos em saúde. Neste contexto, os objetivos do presente estudo são: a) rever a literatura sobre síndrome metabólica, no que diz respeito aos aspectos fisiopatológicos e epidemiológicos envolvendo crianças e jovens; b) discutir o papel da atividade física para a prevenção primária e secundária, bem como a relevância e as dificuldades de atuação do profissional de educação física escolar em seu ambiente de trabalho. Concluímos que a síndrome metabólica tem se intensificado epidemicamente e combater a inatividade física é uma necessidade urgente. Políticas que exaltem a educação física escolar e o seu profissional podem contribuir para a cultivação de hábitos saudáveis e a promoção do ser humano.

Palavras-Chave: Crianças; Jovens; Saúde; Atividade física; Doenças; Obesidade.

\section{METABOLIC SYNDROME AND PHYSICAL EDUCATION}

\begin{abstract}
Metabolic syndrome is the attributed designation to a number of physiological changes in human metabolism which increase dramatically the risk of developing cardiovascular disease and diabetes, that are the major causes of morbidity and mortality today. One of the groups often affected are children and young people of school age. Several epidemiological and biological studies suggest that physical activity can significantly improve this clinical condition, being an important tool for the management and treatment, creating health, less pain and reduced public spending on health. In this context, the objectives of this study are: a) review the literature about metabolic syndrome in relation to pathophysiological and epidemiological studies involving children and young people, b) discuss the role of physical activity for primary and secondary prevention, as well as the
\end{abstract}


importance and difficulties of the physical education teacher in their work environment. We conclude that the metabolic syndrome has been intensified epidemically and combat sedentary lifestyle is an urgent need. Policies that exalt physical education and its professional can contribute to cultivating healthy habits and promoting human being.

Key-Words: Children; Youth; Health; Physical activity; Diseases; Obesity.

\section{SÍNDROME METABÓLICO Y LA EDUCACIÓN FÍSICA ESCOLAR}

\section{RESUMÉN}

El síndrome metabólico es el nombre dado a un conjunto de cambios fisiológicos en el metabolismo humano aumenta dramáticamente el riesgo de desarrollar enfermedades cardiovasculares y la diabetes, las enfermedades que son las principales causas de morbilidad y mortalidad hoy. Uno de los grupos afectados son los niños y jóvenes en edad escolar. Varios estudios epidemiológicos y biológicos sugieren que la actividad física puede mejorar significativamente esta situación clínica, siendo una herramienta importante para el manejo y tratamiento, crear salud, menos dolor y una reducción del gasto público en salud. En este contexto, los objetivos de este estudio son: a) revisar la literatura sobre el síndrome metabólico en relación con los estudios fisiopatológicos y epidemiológicos que afectan a niños y jóvenes, b) discutir el papel de la actividad física para la prevención primaria y secundaria, así como la importancia y las dificultades de la educación física profesional en su entorno de trabajo. Llegamos a la conclusión de que el síndrome metabólico se ha intensificado epidémica y la inactividad física combate es una necesidad urgente. Las políticas que exaltan la educación física y el profesional pueden contribuir a cultivar hábitos saludables y la promoción humana.

Palabras-Clave: Niños; Juventud; Salud; Actividad física; Enfermedades; Obesidad. 


\section{INTRODUÇÃO}

Síndrome metabólica é a designação atribuída a um conjunto de alterações fisiológicas no metabolismo humano que aumenta drasticamente o risco de desenvolver doenças cardiovasculares e o diabetes tipo 2. Essas patologias constituem as principais causas de morbidez e morte nos países desenvolvidos e em desenvolvimento na atualidade. ${ }^{1}$ A projeção para os próximos anos é de que o número de pessoas diagnosticadas com esse tipo de síndrome aumente intensamente nesses países, inclusive entre jovens e crianças, representando uma epidemia em curso e sobrecarregando ainda mais os sistemas públicos de saúde. ${ }^{2-4}$

As modificações culturais e comportamentais de habitantes dos países desenvolvidos e em desenvolvimento têm propiciado o surgimento e aumento da frequência de síndromes e patologias crônicas. O sedentarismo, uma das principais modificações no comportamento do homem moderno, aliado ao estresse descontrolado e a hábitos alimentares com intensa ingestão calórica são aspectos determinantes para o desencadeamento de diversas disfunções fisiológicas e metabólicas. ${ }^{5-6}$

O diagnóstico para síndrome metabólica pode ser facilmente realizado por profissionais da saúde e quanto mais precoce mais eficiente será seu tratamento, podendo prevenir lesões irreversíveis. Dentre os métodos não-invasivos e não-farmacológicos, destacamos a prática de atividade física regular somada à reeducação alimentar. ${ }^{7-11}$ Diversas investigações científicas demonstraram os benefícios da prática regular de atividade física para a saúde, tanto nos aspectos de prevenção de patologias, diminuindo e controlando os fatores de riscos associados a determinadas doenças, como no tratamento de patologias já estabelecidas, sendo uma poderosa ferramenta terapêutica. A prática de exercícios físicos pode beneficiar o sistema cardiovascular, balancear o metabolismo e o perfil lipídico sanguíneo, promover o bem-estar e a saúde mental, aumentar a expectativa e qualidade de vida, por exemplo. ${ }^{12-17}$

Um grande percentual das verbas destinadas ao sistema público de saúde é utilizado no tratamento de doenças cardiovasculares e metabólicas. Por serem, de um modo geral, doenças de longa duração, estão entre as complicações que mais demandam procedimentos 
e serviços de saúde, assim como remédios e hospitalização. ${ }^{18}$ Apesar do alto impacto social e econômico, o investimento ainda é insuficiente para a prevenção dessas patologias, assim como para a educação e promoção da saúde através de atividades físicas para a população em geral. Deveria ser papel do Estado promover a saúde através de atividades que tenham como objetivo conscientizar a população, assim como promover a prática de exercícios físicos, de forma mais expressiva, em regiões e comunidades carentes. ${ }^{19,4,10}$

A educação física escolar possui grande potencial para o trabalho de diversos aspectos relevantes voltados à promoção e educação em saúde, através de seu curso, conteúdos e métodos de ensino. ${ }^{20}$ Ao longo das aulas, o professor tem a possibilidade de propor e discutir atividades associadas à prevenção de patologias e disfunções, conscientizando os alunos sobre comportamentos de risco e fatores ambientais ou modificáveis. ${ }^{7,21-23}$

Entretanto, a carga horária reduzida, a infra-estrutura deficiente na maioria das instituições públicas de ensino, a dificuldade de promover atividades extracurriculares com participação da comunidade por falta de recursos e segurança pública, a falta de educação continuada para os professores da rede pública de ensino, dentre outros fatores, acabam por limitar ações potencialmente benéficas não apenas para alunos da escola, como também para funcionários, parentes e moradores das regiões próximas. ${ }^{24}$

Diante do exposto, os objetivos do presente estudo são: a) rever a literatura sobre síndrome metabólica, no que diz respeito aos aspectos fisiopatológicos e epidemiológicos envolvendo crianças e jovens; b) discutir o papel da atividade física na prevenção primária e secundária, bem como a relevância e dificuldades de atuação do professor de educação física escolar em seu ambiente de trabalho.

\section{REVISÃO DE LITERATURA}

A fundamentação teórica do presente trabalho está dividida em quatro partes: fisiopatologia; diagnóstico; epidemiologia; sedentarismo entre crianças e jovens; benefícios das atividades físicas, e; atividades físicas no ambiente escolar. 


\section{Fisiopatologia}

Das diversas tentativas de se definir a síndrome metabólica, destacamos a estabelecida pela Third Report of the National Cholesterol Education Program Expert Panel on Detection, Evaluation, and Treatment of High Blood Cholesterol in Adults (Adult Treatment Panel III [ATP III]) (National Institutes of Health, 2001). De acordo com esta instituição, uma pessoa para ser diagnosticada como portadora da síndrome deve ter pelo menos três fatores dos seguintes critérios: obesidade abdominal; hipertrigliceridemia; baixo colesterol HDL (lipoproteína de alta densidade); pressão arterial elevada; e alto nível de glicose sanguínea em jejum. . $^{1,25}$

As alterações metabólicas são causadas, principalmente, por sedentarismo, alimentação com excesso de calorias, tabagismo e obesidade ou acúmulo excessivo de gordura abdominal. Diversos estudos demonstram que essas modificações favorecem o desenvolvimento de doenças cardiovasculares, como a insuficiência cardíaca, infarto do miocárdio e doenças coronarianas, podendo levar à morbidez e morte, além de eventos arrítmicos que podem levar à morte súbita cardíaca. A síndrome metabólica também pode favorecer o surgimento de diabetes mellitus tipo 2 e doenças vasculares no sistema circulatório cerebral e periférico. ${ }^{8,26-30}$

A obesidade andróide, excesso de peso corporal com maior depósito de gordura na região abdominal, apresenta uma grande associação com alterações metabólicas e doenças cardiovasculares. Entretanto, o acúmulo de gordura na região abdominal pode ser tanto de gordura visceral intra-abdominal, como subcutânea abdominal, sendo a primeira mais relacionada com alterações metabólicas e doenças cardiovasculares. O método para analisar o exato local de depósito de gordura é extremamente caro e sofisticado, seja a ressonância magnética ou a tomografia computadorizada, dificultando ou impossibilitando seu uso para diagnósticos no sistema público de saúde. No entanto, os dados obtidos através da medição antropométrica da circunferência abdominal, no ponto médio entre o último arco costal e a crista ilíaca, são utilizados com consistência na clínica médica. ${ }^{31-34}$

A resistência à ação da insulina nos tecidos alvos e o nível elevado de insulina plasmática em jejum, características frequentes de indivíduos obesos e com síndrome metabólica, são 
considerados os primeiros sinais do desenvolvimento do diabetes do tipo 2, também conhecido como pré-diabetes. A elevação da concentração do hormônio da insulina e o alto nível glicêmico circulante são considerados fatores desencadeadores de doenças cardiovasculares, além de terem um importante papel no desenvolvimento de outros componentes da síndrome metabólica, como o aumento dos níveis de lipídios e da pressão arterial. ${ }^{35}$ Há algumas décadas, o diabetes tipo 2 era uma doença encontrada majoritariamente em adultos, no entanto, nos últimos anos, tem sido verificado um aumento da prevalência desta doença em crianças e adolescentes, mostrando uma possível corrrelação do aumento da prevalência de obesidade infantil com o desenvolvimento desta doença. $^{22,34-38}$

O hormônio insulina possui diversas funções fisiológicas para o correto controle do metabolismo lipídico, entretanto, em indivíduos obesos e portadores de síndrome metabólica podem ocorrer frequentes alterações no funcionamento de algumas enzimas e no metabolismo lipídico, devido à resistência à insulina. Juntamente com outros fatores endógenos gerados pelas modificações no metabolismo desses indivíduos, podem ocorrer perigosas alterações do perfil lipídico sanguíneo. As principais alterações e com consequências mais alarmantes são: níveis aumentados de LDL (lipoproteína de baixa densidade) devido à maior produção de triglicérides pelo fígado e ao menor catabolismo, redução dos níveis de HDL (lipoptroteína de alta densidade) e aumento de triglicerídeos circulantes. Tais fatores são considerados os maiores causadores de arteriosclerose e doenças coronarianas. ${ }^{31,37-38}$

O aumento da pressão arterial em indivíduos com síndrome metabólica é muito comum, sendo um importante preditor de doenças cardiovasculares. Um dos possíveis mecanismos para se explicar essas alterações é a maior ativação do sistema renina-angiotensinaaldosterona e do sistema nervoso simpático. O ganho excessivo de massa corporal está associado com o aumento da retenção de sódio e expansão do volume extracelular, fatores determinantes para a gênese de hipertensão arterial. ${ }^{39}$ Essas modificações prejudiciais ao organismo humano podem ocorrer em virtude da elevação da atividade da renina plasmática, do angiotensinogênio plasmático, da atividade da enzima conversora de angiotensina e da concentração plasmática de angiotensina dois. Outro fator agravante 
pode ser o aumento do nível de secreção de aldosterona, um hormônio com importante papel na reabsorção de sódio no sistema renal e vasoconstrição periférica. As alterações no sistema nervoso simpático podem estar relacionadas com o aumento da secreção de catecolaminas e outros hormônios, que elevam a pressão arterial, assim como aumentam a frequência cardíaca. ${ }^{26,39}$

As alterações geradas pela síndrome metabólica também são capazes de modificar o perfil trombogênico e inflamatório do organismo. Alguns marcadores bioquímicos, como fibrinogênio e do inibidor do plasminogênio 1, quando apresentam suas concentrações elevadas, aumentam o risco de trombose. Níveis elevados de certos marcadores inflamatórios, como a interleucina 6, o fator de necrose tumoral e a proteína $\mathrm{C}$ reativa, também aumentam a propensão de eventos trombogênicos e processos inflamatórios sistêmicos, elevando ainda mais o risco do desenvolvimento de doença coronariana na vida futura. ${ }^{39-41}$ Estudos recentes demonstram que processos inflamatórios sistêmicos podem aumentar a expressão de marcadores relacionados ao sistema imune inato, como os Toll like receptors. Há correlação entre o aumento da expressão desses marcadores inflamatórios com patologias cardíacas e comprometimento cardiovascular. ${ }^{40}$

\section{Diagnóstico}

O diagnóstico da síndrome metabólica em jovens e crianças ainda é muito controverso: geralmente são considerados os parâmetros utilizados em adultos, entretanto adaptados às idades e estágios de maturação. Crianças com menos de seis anos não devem ser avaliadas no intuito de se diagnosticar a síndrome, pois podem apresentar parâmetros muito questionáveis. Em crianças de 6 a 10 anos, apesar de questionamentos consistentes, a avaliação deve focar no acúmulo de gordura abdominal. Considera-se tolerável a circunferência abdominal acima de 90 centímetros. Além disso, a verificação do perfil sanguíneo e da pressão arterial também é fundamental. ${ }^{42}$ Para o diagnóstico de jovens entre 10 e 16 anos já existem parâmetros bem definidos e utilizados na clínica médica: glicemia, após 12 horas de jejum, acima de $100 \mathrm{mg} / \mathrm{dl}$, triglicerídeos acima de $150 \mathrm{mg} / \mathrm{dl}$, HDL abaixo de $40 \mathrm{mg} / \mathrm{dl}$, assim como pressão sanguínea de $130 \mathrm{mmHg}$ (sistólica) e $85 \mathrm{mmHg}$ (diastólica), e circunferência abdominal acima de 90 centímetros. A partir dos 16 anos, são seguidos os modelos para adultos: obesidade abdominal (circunferência da cintura de 102 
cm nos homens e $88 \mathrm{~cm}$ nas mulheres), hipertrigliceridemia (níveis acima de $150 \mathrm{mg} / \mathrm{dl}$ ), baixo colesterol HDL (níveis abaixo de $40 \mathrm{mg} / \mathrm{dl}$ em homens e $50 \mathrm{mg} / \mathrm{dl}$ em mulheres), pressão arterial elevada (sistólica acima de $130 \mathrm{mmHg}$ e diastólica acima $85 \mathrm{mmHg}$ ) e alto nível de glicose de jejum (acima de $110 \mathrm{mg} / \mathrm{dl}$ ). ${ }^{8,43}$

Infelizmente, muitos jovens não têm acesso periódico a consultas ou são neglicenciados pelo profissional da saúde. Consequentemente, tanto o diagnóstico quanto o tratamento não ocorrem de forma precoce e a realidade caótica dos sistemas de saúde tende a piorar cada vez mais.

\section{Epidemiologia}

A Epidemiologia é uma ciência de extrema importância para a sociedade, pois estuda a ocorrência, a distribuição e os fatores determinantes dos eventos relacionados à saúde da população. Através dos dados obtidos por métodos de pesquisa epidemiológicos, medidas podem ser propostas para controlar a saúde, por exemplo, de forma mais eficiente e econômica. Entretanto, infelizmente, o Brasil ainda carece de estudos epidemiológicos que avaliam a prevalência da síndrome metabólica entre crianças e adolescentes, embora existam projeções alarmantes para o futuro. ${ }^{31-32,39}$

Nos últimos 40 anos, houve uma grande mudança nos hábitos alimentares no Brasil. Comparando uma pesquisa realizada em 1974, pelo Estudo Nacional da Despesa Familiar, com os dados da Pesquisa sobre Padrões de Vida em 1996, nota-se um aumento na população infantil e de adolescentes que estão acima do peso ou obesos, de 4,1 para $13,9 \% .{ }^{31-32}$ Um estudo realizado no Estado de São Paulo avaliou a prevalência da síndrome metabólica em jovens com sobrepeso. A partir de um grupo com média de aproximadamente 10 anos de idade concluiu-se que a frequência dessa síndrome é elevada, tendo uma prevalência de $42 \%$ dos jovens avaliados. O grupo concluiu ainda, que dentre os componentes utilizados para o diagnóstico, foram observadas com maior frequência a circunferência abdominal e a pressão arterial elevadas. ${ }^{44}$ Estudos recentes realizados nos Estados Unidos mostram que nos últimos 20 anos o número de crianças e adolescentes com sobrepeso triplicou. Segundo estimativas recentes da população entre dois e 19 anos, $17,1 \%$ se encontram acima do peso e $32,2 \%$ dos jovens com 20 anos são obesos. ${ }^{45} \mathrm{O}$ 
número de estudos realizados com o objetivo de quantificar a prevalência da síndrome metabólica em crianças e adolescentes, entretanto, é menor que para os demais grupos. China, Coréia do Sul, México, Irã, Índia, Turquia, Estados Unidos e Itália, possuem um total de 16 estudos que preenchem os critérios adotados pela Organização Mundial de Saúde e pela National Cholesterol Education Programs. ${ }^{46}$

Em 1999, de acordo com a National Center for Health Statistics, a prevalência da síndrome em adolescentes de 12 a19 anos era de aproximadamente 9,4\%, representando cerca de 2,9 milhões de pessoas nos Estados Unidos. Quatro anos após, esse percentual teria subido para 10\%, em de um estudo epidemiológico com uma amostra de 3400 crianças publicado na principal revista de cardiologia do mundo. ${ }^{47}$ Os dados estatísticos referentes a incidência da síndrome metabólica entre jovens adultos (20 a 29 anos), adultos, e idosos a partir de 60 anos são alarmantes. ${ }^{4,39,48}$

\section{Sedentarismo entre crianças e jovens}

O termo sedentarismo foi utilizado inicialmente para descrever o processo de transição cultural da colonização nômade para o permanente. Como termo biomédico, é denominado como estilo de vida com baixa ou nenhuma prática de exercícios físicos ou desportiva. $\mathrm{O}$ sedentarismo é uma das principais modificações culturais e comportamentais do século e é uma das principais causas de morte evitáveis no mundo. ${ }^{49-50}$

As complicações geradas pelo sedentarismo são diversas e há inúmeras evidências de que a inatividade física na infância e juventude determina o surgimento de doenças. ${ }^{22,27,30,51-52}$

Sua prevalência é a alta em todo o mundo, e a projeção para os próximos anos também é alarmante. Na Europa, o sedentarismo varia entre $43.3 \%$ (Suécia) e $87.8 \%$ (Portugal). ${ }^{53}$ Nos Estados Unidos, entre jovens adultos a inatividade física é de $35 \%$ e com o avanço da idade este percentual aumenta, chegando a $75 \%$ em idosos. O Brasil não fica atrás e acompanha a tendência da Europa e Estados Unidos. ${ }^{54}$

Entre crianças e jovens o aumento na prevalência do sedentarismo também tem aumentado ao longo dos últimos anos, assim como sua projeção para os próximos anos. Em um estudo 
desenvolvido no Rio Grande do Sul, envolvendo 4.452 participantes de 10 a 12 anos de idade, a prevalência de sedentarismo foi de $58,2 \%$, usando como parâmetro a prática de 300 minutos por semana de atividade física. No município de Niterói (RJ), 85\% dos meninos e $94 \%$ das meninas foram classificadas como sedentários. ${ }^{35}$

\section{Benefícios das atividades físicas}

Parece que o ser humano não se desenvolveu ao longo da evolução das espécies para sobreviver de forma fisicamente inativa. ${ }^{55} \mathrm{~A}$ inatividade física parece inibir a produção de diversas proteínas que atuam em mecanismos promotores de saúde e aumentar a síntese de proteínas que promovem doenças. ${ }^{56}$ Esses mecanismos estão associados, por exemplo, com distúrbios no metabolismo, redução da produção de mitocôndrias, indução do estresse oxidativo, diminuição da capacidade de reparo tecidual, menor sinalização para formação de novos neurônios, sinapses e vasos sanguíneos cerebrais. Consequentemente, tornam-se eminentes patologias como o diabetes tipo $2,{ }^{57-58}$ doenças neurodegenerativas - que são consideradas por alguns autores como o diabetes do cérebro ou tipo $3^{59-60}$ - depressão e transtornos de humor e ansiedade. ${ }^{61} \mathrm{O}$ estresse oxidativo, caracterizado pelo aumento desproporcional na concentração de radicais livres em comparação com espécies antioxidantes, gera danos em estruturas protéicas fundamentais à homeostase celular, comprometendo sua saúde e sobrevivência. ${ }^{55}$

Felizmente, estímulos como os proporcionados pelas atividades físicas são suficientes para ativar genes que promovem a saúde em diversos aspectos. ${ }^{56} \mathrm{O}$ sistema bioenergético tornase mais equilibrado. O perfil lipídico passa a apresentar menor quantidade de triglicerídeos circulantes através do aumento na concentração de lipoproteínas de alta densidade e redução na concentração de lipoproteínas de baixa densidade. ${ }^{8,22,39,62}$ Os tecidos melhoram a capacidade em captar glicose, refletindo sobre o controle do perfil glicêmico. ${ }^{57-58} \mathrm{O}$ sistema cardiovascular é fortalecido, diminuindo a probabilidade de lesões e cardiomiopatias. O tecido cardíaco torna-se mais apto a consumir oxigênio e produzir energia. Há um aumento da circulação microvascular periférica, evitando microvasculopatias e aterosclerose. ${ }^{51} \mathrm{O}$ sistema parassimpático e simpático do sistema nervoso autonômico cardíaco equilibra-se e consequências deletérias ao sistema cardiovascular são evitadas ${ }^{16}$. A quantidade de ingestão de fármacos pode ser diminuída 
através da prática regular de atividades físicas, incluindo a dependência de insulina ${ }^{10 ; 63}$. O aumento da massa isenta de gordura e a redução do acúmulo de gordura abdominal ajudam no combate contra a obesidade infantil. Há relatos na literatura de que jovens sedentários, obesos ou acima do peso, após iniciarem práticas desportivas, experimentam benefícios psicossociais, como a melhora da auto-estima, companheirismo, bem-estar psicológico, ampliação do círculo social, entre outros. ${ }^{64-65}$

\section{Atividades físicas no ambiente escolar}

É papel da educação física escolar criar e aplicar programas de prevenção e tratamento da saúde. Além de prescrever e acompanhar a prática de exercícios físicos e desportivos, o professor de educação física pode conscientizar seus alunos não apenas sobre os benefícios de uma vida ativa fisicamente, como também sobre outros componentes ambientais favoráveis à vida. O professor de educação física escolar tem um papel crucial no controle da síndrome metabólica e de outras desordens crônicas e é um elemento essencial à saúde coletiva e pública.

É comum observarmos que alunos com peso excessivo não participam efetivamente das aulas de educação física. Este problema pode ser ocasionado pela própria auto-segregação do aluno, que não se sente à vontade, não possui habilidades ou interesse nas atividades propostas. Com frequência são alvos de bullying e sofrem de complicações psicossociais, como sentimentos de inferioridade e limitação, por exemplo. ${ }^{66-67}$

Alunos com síndrome metabólica podem participar das aulas propostas no ambiente escolar, desde que respeitadas suas limitações. $\mathrm{O}$ foco deve ser sempre o aluno e nunca a doença. As atividades devem contemplar componentes da aptidão física, como a força, resistência muscular localizada, flexibilidade, aptidão cardiorrespiratória, equilíbrio, agilidade e consciência corporal, por exemplo. Isso não quer dizer que o prazer e a motivação não sejam desconsiderados, muito pelo contrário. $\mathrm{O}$ ideal seria a realização das aulas diariamente, mas o mínimo de três vezes por semana não deve ser descartado. ${ }^{65,68 \text {, }}$ 69,70 Algumas modificações na aparência e no comportamento do aluno podem ser observadas durante a prática como a palidez, hálito cetônico, tontura, sudorese excessiva, náuseas, vista turva e desequilíbrio. O professor deve estar constantemente atento a essas 
alterações, podendo evitar eventuais acidentes ou choques. Ao perceber algum desses sinais, a atividade intensa deve ser interrompida visando a recuperação do ritmo homeostático de repouso. ${ }^{17,39,62,71-72}$

Apesar das diversas formas de atuação existentes e do enorme conhecimento para embasar tais atividades, diferentes fatores podem dificultar ou até mesmo impedir a atuação do profissional. A educação física escolar dispõe de uma carga horária reduzida, sendo realizada muitas vezes em apenas um tempo de 50 minutos por semana; há uma desproporção acentuada entre o número de profissionais e a quantidade de alunos, fazendo com que as turmas sejam superlotadas; a estrutura física e material, principalmente nas instituições públicas, é precária; a carência de segurança pública e de recursos financeiros pode inviabilizar a realização de atividades extracurriculares que estimulem alunos e a comunidade adjacente; não há incentivo à educação continuada.

\section{CONCLUSÃO}

Podemos concluir que a síndrome metabólica tem se intensificado epidemicamente e combater a inatividade física é uma necessidade urgente. A educação física escolar deve ser considerada uma importante ferramenta a favor da educação em saúde, cultivação de hábitos saudáveis e promoção do ser humano. Para exaltá-la, são necessárias políticas que facilitem a atuação do profissional.

\section{REFERÊNCIAS}

${ }^{1}$ ALBERTI, K. G. et al. The metabolic syndrome: a new worldwide definition. Lancet, London, v. 366, p. 1059-1062, 2005.

${ }^{2}$ SEKI, M.; MATSUO, T.; CARRILHO, A. J. Prevalence of metabolic syndrome and associated risk factors in brazilian schoolchildren. Public Health Nutrition, v. 12, n. 7, p. 947-952, july 2009.

${ }^{3}$ CHEN, W.; BERENSON, G. S. Metabolic syndrome: definition and prevalence in children. Jornal de Pediatria, Rio de Janeiro, v. 83, n. 1, 2007. 
${ }^{4}$ SCHOLZE, J. et al. Epidemiological and economic burden of metabolic syndrome and its consequences in patients with hypertension in germany, spain and italy; a prevalence-based model. BMC Public Health, v.10, p. 529, 2010.

${ }^{5}$ SALMON, J.; TIMPERIO, A. Prevalence, trends and environmental influences on child and youth physical activity. Medicine and Sport Science, v. 50, p.183-199, 2007.

${ }^{6}$ HASLAM, D. W.; JAMES, W. P. Obesity. Lancet, London, v. 366, p.1197-1209, 2005.

${ }^{7}$ BRANDÃO, A. P. et al. Management of metabolic syndrome in young population. American Journal of Therapy, v.15, p. 356-361, 2008.

${ }^{8}$ HALPERN, A. et al. Metabolic syndrome, dyslipidemia, hypertension and type 2 diabetes in youth: from diagnosis to treatment. Diabetology \& Metabolic Syndrome, $\mathrm{n}$. 2, p. $55,2010$.

${ }^{9}$ LEÃO, L. S. Nutritional interventions in metabolic syndrome: a systematic review. Arquivos Brasileiros de Cardiologia, São Paulo, v. 97, n. 3, p. 260-265, 2011.

${ }^{10}$ MISIGOJ-DURAKOVIĆ, M.; DURAKOVIĆ, Z. The early prevention of metabolic syndrome by physical exercise. Collegium Antropologicum, v. 33, n. 3, p. 759-764, 2009.

${ }^{11}$ OSHIDA, Y. Effects of exercise-training on metabolic syndrome. Nihon Rinsho, v. 64, suppl 9, p. 584-588, 2006.

${ }^{12}$ MCCALL, A.; RAJ, R. Exercise for prevention of obesity and diabetes in children and adolescents. Clinics in Sports Medicine, v. 28, p. 393-421, 2009.

${ }^{13}$ NAGATOMO, F. et al. The effects of running exercise on oxidative capacity and pgc-1 $\alpha$ mrna levels in the soleus muscle of rats with metabolic syndrome. Journal of Physiology Science, v. 62, n. 2, p. 105-114, 2012. 
${ }^{14}$ PESCATELLO, L. S. et al. The metabolic syndrome and the immediate antihypertensive effects of aerobic exercise: a randomized control design. BMC Cardiovascular Disorders, v. 8, p. 12, 2008.

${ }^{15}$ POTTEIGER, J. A. et al. Resistance exercise and aerobic exercise when paired with dietary energy restriction both reduce the clinical components of metabolic syndrome in previously physically inactive males. European Journal of Applied Physiology, Heidelberg, v. 112, n. 6, p. 2035-2044, 2012.

${ }^{16}$ SOARES-MIRANDA, L. et al. Metabolic syndrome, physical activity and cardiac autonomic function. Diabetes Metabolism Research and Reviews, 2012

${ }^{17}$ SVACINOVÁ H. Exercise therapy for patients with metabolic syndrome. Vnitriní Lékarstvi, v. 53, n. 5, p. 540-544, 2007.

${ }^{18}$ AZAMBUJA, M. I. et al. Economic burden of severe cardiovascular diseases in Brazil: an estimate based on secondary data. Arquivos Brasileiros de Cardiologia, São Paulo, v. 91, n. 3, p. 148-155, p. 163-171, 2008.

${ }^{19}$ BIELEMANN, R.; KNUTH, A.; HALLAL, P. Atividade física e redução de custos por doenças crônicas ao sistema único de saúde; physical activity and cost savings for chronic diseases to the sistema único de saúde. Revista Brasileira de Atividade Física \& Saúde, Londrina, v. 15, n.1, p. 9-14, 2010.

${ }^{20}$ ORFEI, J. M.; TAVARES, V. P. Promoção da saúde na escola através das aulas de Educação Física. Campinas: Ed. da Unicamp, 2007.

${ }^{21}$ DAMIÃO, R. et al. Impact of a lifestyle intervention program on metabolic, anthropometrical and dietary profile of Japanese-Brazilians with and without metabolic syndrome. Arquivos Brasileiros de Endocrinologia e Metabologia, São Paulo, v. 55, n. 2, p. 134-145, mar. 2011.

Conexões: revista da Faculdade de Educação Física da UNICAMP, Campinas, v. 11, n. 1, 124-143, jan./mar. 2013.137 ISSN: 1983-9030 
${ }^{22}$ GUINHOUYA, B. C. et al. Evidence of the influence of physical activity on the metabolic syndrome and/or on insulin resistance in pediatric populations: a systematic review. International Journal Pediatric and Obesity, v. 6, n. 5-6, p. 361-388, oct. 2011.

${ }^{23}$ JAGO, R. et al. Healthy intervention: fitness, physical activity, and metabolic syndrome results. Medicine and Science in Sports and Exercise, Hagerstown, v. 43, n. 8, p. 15131522, aug. 2011.

${ }^{24}$ MUSTAFA, Z. H. A Educação Física e a educação para a saúde nas escolas públicas da cidade de Dourados/MS. 2006. 188f. Dissertação (Mestrado em Ciências da Saúde) Universidade de Brasília, Brasília, 2006.

${ }^{25}$ CAGAMPANG, F. R.; POORE, K. R.; HANSON, M. A. Developmental origins of the metabolic syndrome: body clocks and stress responses. Brain Behavior and Immunity, Orlando, v. 25, n. 2, p. 214-220, feb. 2011.

${ }^{26}$ CAMPANA, E. M. et al. Blood pressure in young individuals as a cardiovascular risk marker: the Rio de Janeiro study. Arquivos Brasileiros de Cardiologia, São Paulo, v. 93, n. 6, p. 608-615, p. 657-665, dec. 2009.

${ }^{27}$ GRUNDY, S. M. et al. Cardiorespiratory fitness and metabolic risk. American Journal of Cardiology, New York, v. 109, p. 988-993, jan. 2012.

${ }^{28}$ RODRIGUES, A. N. et al. Cardiovascular risk factors, their associations and presence of metabolic syndrome in adolescents. Jornal de Pediatria, Rio de Janeiro, v. 85, n. 1, p. 55$60,2009$.

${ }^{29}$ SCHROEN, B.; HEYMANS, S. Small but smart--microRNAs in the centre of inflammatory processes during cardiovascular diseases, the metabolic syndrome, and ageing. Cardiovascular Research, London, nov. 2011. 
${ }^{30}$ STABELINI NETO, A. et al. Physical activity, cardiorespiratory fitness, and metabolic syndrome in adolescents: a cross-sectional study. BMC Public Health, v. 11, p. 674, 2011.

${ }^{31}$ FERREIRA, A. P.; NÓBREGA, O. E. T.; FRANÇA, N. M. Association of body mass index and insulin resistance with metabolic syndrome in Brazilian children. Arquivos Brasileiros de Cardiologia, São Paulo, v. 93, n. 2, p. 147-153, aug. 2009.

${ }^{32}$ FERREIRA, A. P. et al. Prediction of metabolic syndrome in children through anthropometric indicators. Arquivos Brasileiros de Cardiologia, São Paulo, v. 96, n. 2, p. 121-125, feb. 2011.

${ }^{33}$ FONSECA, F. L. et al. Overweight and cardiovascular risk among young adults followed-up for 17 years: the Rio de Janeiro study, Brazil. Arquivos Brasileiros de Cardiologia, São Paulo, v. 94, n. 2, p. 193-201, p. 207-215, p.196-204, feb. 2010.

${ }^{34}$ WEISS, R. et al. Obesity and the metabolic syndrome in children and adolescents. New England Journal of Medicine, Waltham, v. 350, n. 23, p. 2362-2374, jun. 2004.

${ }^{35}$ ALVAREZ, M. M. et al. Insulin resistance in Brazilian adolescent girls: association with overweight and metabolic disorders. Diabetes Research and Clinical Practice, Amsterdam, v. 74, n. 2, p. 183-188, nov. 2006.

${ }^{36}$ SILVA, R. C. et al. Metabolic syndrome and insulin resistance in normal glucose tolerant brazilian adolescents with family history of type 2 diabetes. Diabetes Care, Alexandria, v. 28, n. 3, p. 716-718, mar. 2005.

${ }^{37}$ STRUFALDI, M. W.; DA SILVA, E. M.; PUCCINI, R. F. Insulin resistance among Brazilian schoolchildren: association with risk factors for cardiovascular diseases. Acta Paediatrica, Oslo, v. 98, n. 10, p. 1646-1650, oct. 2009. 
${ }^{38}$ SUNG, K. C. et al. Elevated fasting insulin predicts the future incidence of metabolic syndrome: a 5-year follow-up study. Cardiovascular Diabetology, v. 10, p. 108, 2011.

${ }^{39}$ AIZAWA, K. et al. Effects of lifestyle modification on central artery stiffness in metabolic syndrome subjects with pre-hypertension and/or pre-diabetes. Diabetes Research and Clinical Practice, Amsterdam, v. 83, n. 2, p. 249-256, feb. 2009.

${ }^{40}$ JIALAL, I. et al. Increased toll-like receptor activity in patients with metabolic syndrome. Diabetes Care, Alexandria, feb. 2012.

${ }^{41}$ PARRETT, A. L. et al. Adiposity and aerobic fitness are associated with metabolic disease risk in children. Applied Physiology Nutrition and Metabolism, v. 36, n. 1, p. 72-79, feb. 2011.

${ }^{42}$ PERGHER, R. N. et al. Is a diagnosis of metabolic syndrome applicable to children? Jornal de Pediatria, Rio de Janeiro, v. 86, n. 2, p. 101-108, mar./apr. 2010.

${ }^{43}$ MANCINI, M. C. Metabolic syndrome in children and adolescents: criteria for diagnosis. Diabetol and Metabolic Syndrome, v. 1, n. 1, p. 20, 2009.

${ }^{44}$ BUFF, C. D. G. et al. Freqüência de síndrome metabólica em crianças e adolescentes com sobrepeso e obesidade. Revista Paulista de Pediatria, São Paulo, v. 25, n. 3, p. 221226, set. 2007.

${ }^{45}$ OGDEN, C. L. et al. Prevalence of obesity and trends in body mass index among US children and adolescents, 1999-2010. JAMA, Chicago, v. 307, n. 5, p. 483-490, feb. 2012.

${ }^{46}$ MORAES, A. C. et al. Prevalence of metabolic syndrome in adolescents: a systematic review. Cadernos de Saude Pública, Rio de Janeiro, v. 25, n. 6, p. 1195-1202, jun. 2009. 
${ }^{47}$ DE FERRANTI, S. D. et al. Prevalence of the metabolic syndrome in American adolescents: findings from the Third National Health and Nutrition Examination Survey. Circulation, Baltimore, v. 110, n. 16, p. 2494-2497, oct. 2004.

${ }^{48}$ SCHWANDT, P. et al. A three-country study on the components of the metabolic syndrome in youths: the BIG study. International Journal of Obesity, Washington, v. 5, n. 4, p. 334-341, aug. 2010.

${ }^{49}$ WORLD HEALTH ORGANIZATION (WHO). Physical inactivity: a global public health problem. Washington, 2008.

${ }^{50}$ MICHAUD, C. M.; MURRAY, C. J.; BLOOM, B. R. Burden of disease: implications for future research. JAMA, Chicago, v. 285, n. 5, p. 535-539, feb. 2001.

${ }^{51}$ ANDERSEN, L. B. et al. Physical activity and cardiovascular risk factors in children. British Journal of Sports Medicine, Loghborough, v. 45, n. 11, p. 871-876, sept. 2011.

${ }^{52}$ LEE, D. C. et al. Changes in fitness and fatness on the development of cardiovascular disease risk factors hypertension, metabolic syndrome, and hypercholesterolemia. Journal of the American College of Cardiology, New York, v. 59, n. 7, p. 665-672, feb. 2012.

${ }^{53}$ VARO, J. J. et al. Distribution and determinants of sedentary lifestyles in the European Union. Internatoional Journal of Epidemiology, Oxford, v. 32, n. 1, p. 138-146, feb. 2003.

${ }^{54}$ PITANGA, F. J. G. Prevalência e fatores associados ao sedentarismo no lazer em adultos. Cadernos de Saúde Pública, Rio de Janeiro, v. 21, n. 3, p. 870-877, 2005.

${ }^{55}$ RADAK, Z.; CHUNG, H. Y.; GOTO, S. Systemic adaptation to oxidative challenge induced by regular exercise. Free Radical Biology \& Medicine, New York, v. 44, n. 2, p. 153-159, jan. 2008.

Conexões: revista da Faculdade de Educação Física da UNICAMP, Campinas, v. 11, n. 1, 124-143, jan./mar. 2013.141 ISSN: 1983-9030 
${ }^{56}$ BOOTH, F. W.; CHAKRAVARTHY, M. V.; SPANGENBURG, E. E. Exercise and gene expression: physiological regulation of the human genome through physical activity. Journal of Physiology, Cambridge, v. 543, n. pt 2, p. 399-411, sept. 2002.

${ }^{57}$ SALTIEL, A. R. New perspectives into the molecular pathogenesis and treatment of type 2 diabetes. Cell, Cambridge, v. 104, n. 4, p. 517-529, feb. 2001.

${ }^{58}$ ROPELLE, E. R. et al. Acute exercise modulates the Foxo1/PGC-1alpha pathway in the liver of diet-induced obesity rats. Journal of Physiology, Cambridge, v. 587, n. pt. 9, p. 2069-2076, may 2009.

${ }^{59}$ DE LA MONTE, S. M. Insulin resistance and Alzheimer's disease. BMB Reports, Korea, v. 42, n. 8, p. 475-481, aug. 2009.

${ }^{60}$ DE FELICE, F. G. et al. Protection of synapses against Alzheimer's-linked toxins: insulin signaling prevents the pathogenic binding of abeta oligomers. Proceedings of the National Academy of Sciences of the United States of America, v. 106, n. 6, p. 19711976, feb. 2009.

${ }^{61}$ DESLANDES, A. et al. Exercise and mental health: many reasons to move. Neuropsychobiology, Switzerland, v. 59, n. 4, p. 191-198, 2009.

${ }^{62}$ SATO, Y. et al. Clinical aspects of physical exercise for diabetes/metabolic syndrome. Diabetes Research and Clinical Practice, v. 77, suppl 1, p. S87-91, sept. 2007.

${ }^{63}$ KIM, Y.; LEE, S. Physical activity and abdominal obesity in youth. Applied Physiology, Nutrition and Metabolism, v. 34, n. 4, p. 571-581, aug 2009.

${ }^{64}$ NEUMANN, N. U.; FRASCH, K. Coherences between the metabolic syndrome, depression, stress and physical activity. Psychiatrische Praxis, v. 36, n. 3, p. 110-114, apr. 2009.

Conexões: revista da Faculdade de Educação Física da UNICAMP, Campinas, v. 11, n. 1, 124-143, jan./mar. 2013. ISSN: 1983-9030 
${ }^{65}$ HOLMES, M. E. et al. Physical activity, stress, and metabolic risk score in 8- to 18-yearold boys. Journal Phys Act Health, v. 5, n. 2, p. 294-307, mar. 2008.

${ }^{66}$ MORRIS, H. H. The role of school physical education in public health. Research Quarterly and Exercise in Sport, v. 62, n. 2, p. 143-147, jun. 1991.

${ }^{67}$ TORRES, H. D. C.; HORTALE, V. A.; SCHALL, V. A experiência de jogos em grupos operativos na educação em saúde para diabéticos. Cadernos de Saúde Pública, Rio de Janeiro, v. 19, n. 4, p. 1039-1047, ago. 2003.

${ }^{68}$ COPPEN, A. M.; RISSER, J. A.; VASH, P. D. Metabolic syndrome resolution in children and adolescents after 10 weeks of weight loss. Journal Cardiometab Syndr, v. 3, n. 4, p. 205-210, 2008.

${ }^{69}$ GUINHOUYA, B. C. Physical activity in preventing metabolic syndrome in children. Medicine Science, Paris, v. 25, n. 10, p. 827-833, oct. 2009.

${ }^{70}$ MICHELI, L. et al. Fitness and health of children through sport: the context for action. British Journal of Sports Medicine, Loughborough, v. 45, n. 11, p. 931-936, sept. 2011.

${ }^{71}$ SUBIRATS BAYEGO, E.; SUBIRATS VILA, G.; SOTERAS MARTÍNEZ, I. Exercise prescription: indications, dosage and side effects. Medicina Clinica, Barcelona, v. 138, n. 1, p. 18-24, jan. 2012.

${ }^{72}$ TANAKA, K. et al. Exercise prescription for people with metabolic syndrome. Nihon Rinsho: japanese journal of clinical medicine, v. 64, suppl. 9, p. 574-579, dec. 2006.

Recebido em: 04 jul. 2012 Aceito em: 17 jan. 2013

Contato: Guillermo Esteban Gutiérrez Lalcote ggtie65@ hotmail.com

Conexões: revista da Faculdade de Educação Física da UNICAMP, Campinas, v. 11, n. 1, 124-143, jan./mar. 2013.143 ISSN: 1983-9030 